\title{
INTEGRANDO LA FORMACIÓN DE PUBLICIDAD EN RADIO EN EL CONTEXTO PROFESIONAL
}

\section{INTEGRATING RADIO ADVERTISING COURSES IN A PROFESSIONAL CONTEXT}

\section{AUTORES}

Emma Rodero: Profesora Titular de Comunicación Audiovisual y Publicidad. Facultad de Comunicación. Universidad Pompeu Fabra. Barcelona (España). emma.rodero@upf.edu

Olatz Larrea: Asistente de docencia de Comunicación Audiovisual y Publicidad. Facultad de Comunicación. Universidad Pompeu Fabra. Barcelona (España). olatz.larrea@upf.edu

Marina Vázquez: Asistente de docencia de Comunicación Audiovisual y Publicidad. Facultad de Comunicación. Universidad Pompeu Fabra. Barcelona (España). marina.vazquez@upf.edu

\section{RESUMEN}

Desde todos los sectores se coincide en afirmar que la calidad publicitaria de la radio se encuentra muy por debajo de la de otros medios de comunicación. La explicación principal que apuntan muchos autores a esta crisis de la publicidad en radio es precisamente la débil formación universitaria en esta materia. Esto significa que no estamos formando adecuadamente profesionales en una especialidad que comporta 
la sensibilización y el dominio de un lenguaje propio y, por lo tanto, el saber trabajar con criterios de calidad, usando las posibilidades que ofrecen las nuevas tecnologías. Este texto da cuenta de la propuesta de un proyecto que pretende plantear una solución interdisciplinar y transversal a la crisis del sector a través de una metodología, una estructura y una dinámica acorde con uno de los principios básicos del Espacio Europeo de Enseñanza Superior: la adquisición de las competencias necesarias para que los estudiantes sean capaces de desarrollarse en el contexto profesional de forma eficiente, autónoma y flexible mediante una metodología de enseñanza centrada en la participación activa del estudiante (learning by doing).

\title{
PALABRAS CLAVE
}

Publicidad - Radio - EEES - Contexto profesional - Nuevas tecnologías

\begin{abstract}
For many authors, the quality of radio advertising is far below the other audiovisual media. The main explanation to the crisis of radio advertising belongs to the weak training about this area in the university, as we are not properly forming good professionals in this topic. This training involves the knowledge of the radio language and, therefore, the ability to work with quality standards, using the possibilities offered by the new technologies. Therefore, this text explains a project that tries to contribute with an interdisciplinary solution to the crisis in this sector. It is based on a methodology, structure and dynamic according to one of the basic principles of the European Higher Education: the acquisition of the skills that the students require to be able to manage in the professional context in an efficient,
\end{abstract}


autonomous and flexible way. In these sense, the methodology focuses on active student participation (learning by doing).

\section{KEY WORDS}

Advertising - Radio - EEES - Professional context - New technologies

\section{ÍNDICE}

1. Justificación del proyecto

2. Objetivos del proyecto

3. Elementos pedagógicos del proyecto

4. Desarrollo del proyecto

5. Evaluación del proyecto

6. Ventajas del proyecto

7. Resultados e impacto del proyecto

8. Conclusiones y líneas de trabajo

9. Bibliografía

\section{Justificación del proyecto}

Desde todos los sectores se coincide en afirmar que la calidad publicitaria de la radio se encuentra muy por debajo de la de otros medios de comunicación. Desde hace muchos años los contenidos se consideran pobres y escasamente creativos en comparación con las producciones para televisión (Rodero, 2008). La explicación principal que apuntan muchos autores a esta crisis de la publicidad en radio es precisamente la débil formación universitaria en esta materia. 
"Los jóvenes creativos (publicitarios) no conocen la radio. Al menos un buen porcentaje de ellos. El aluvión de planificadores con poca experiencia los ubica frente a una terminal de ordenador, como simples sacadores de datos. $Y$ como consecuencia de ello la radio siempre sale perjudicada." (García, 2008).

Esto significa que no estamos formando adecuadamente profesionales en una especialidad que comporta la sensibilización y el dominio de un lenguaje propio y, por lo tanto, el saber trabajar con criterios de calidad, usando las posibilidades que ofrecen las nuevas tecnologías. Ante esta situación, se hace necesario reconvertir la formación de estos futuros profesionales diseñando y aplicando una nueva estrategia docente sobre esta materia que facilite la adquisición de las competencias propias de las crecientes necesidades y perspectivas del desarrollo de la actividad publicitaria en radio acorde siempre con uno el Espacio Europeo de Enseñanza Superior (EEES).

Uno de los principios fundamentales del EEES se centra en la adquisición de las competencias necesarias para los estudiantes sean capaces de desarrollarse en el contexto profesional de forma eficiente, autónoma y flexible mediante una metodología de enseñanza centrada en la participación activa del estudiante (learning by doing), una idea presente ya en el pensamiento clásico por su eficiencia demostrada: "me lo dijeron y lo olvidé, lo vi y lo entendí, lo hice y lo aprendi" (Confucio). Sobre este principio básico, este proyecto, aplicado a la asignatura Producción y Realización de Publicidad en Radio del grado de Publicidad y Relaciones Públicas de la UPF, pretende desarrollar un método de aprendizaje guiado basado en la adquisición de conocimientos y habilidades orientados a la consecución de resultados dirigidos a la práctica profesional aplicando, a este fin, las nuevas tecnologías. La formación por competencias profesionales que se pretende aplicar desemboca necesariamente en la determinación de perfiles profesionales concretos, adecuados al mercado laboral y a la demanda de la industria de la comunicación 
publicitaria (Aneca, 2005) así como responder a las nuevas habilidades tecnológicas de los estudiantes.

Por tanto, a lo largo de las siguientes páginas, se describe un proyecto que pretende plantear una solución interdisciplinar y transversal a la crisis del sector a través de una metodología, una estructura y una dinámica integradora con las necesidades del contexto profesional, a través del desarrollo de prácticas de actuación laboral, basadas en la interrelación sistémica de competencias académicas, profesionales y analíticas, utilizando métodos productivos y científicos con base en la solución de problemas y mediante el uso de las nuevas tecnologías.

"La educación basada en competencias es la creación de oportunidades para los estudiantes y los trabajadores, cerca de su mundo de experiencia en un ambiente de aprendizaje significativo (preferible la práctica profesional) en donde el alumno puede desarrollar sistemas integrados, orientados al rendimiento las capacidades para manejar los problemas en la práctica" (Wesselink et al., 2003).

\section{Objetivos del proyecto}

Este proyecto tiene como principal finalidad reconvertir una materia docente en una disciplina integradora con el contexto profesional, dotando a los alumnos de una visión real y crítica de la situación de la publicidad en el medio radiofónico, a través de la adquisición de competencias profesionales y tecnológicas. El método de aprendizaje guiado está basado en la realización de proyectos. Los objetivos específicos que se pretenden lograr se derivan de la aplicación de cuatro principios pedagógicos esenciales del Espacio Europeo de Enseñanza Superior: 
1. Principio de identificación y resolución de problemas profesionales reales. La finalidad de este principio es que los estudiantes asuman un papel activo, comprometido y creador, demostrando habilidades para la toma de decisiones oportunas y para la solución de problemas en los diversos campos de la producción y realización de publicidad en radio. De esta manera, los futuros profesionales se convierten en planificadores de dos campañas publicitarias basadas en necesidades y datos de clientes reales. El proceso docente va dirigido a la solución de problemas profesionales reales de la industria radiofónica.

2. Principio de consecución de resultados dirigidos a la práctica profesional. El objetivo se centra aquí en aportar resultados innovadores y originales que respondan a las crecientes necesidades de la industria radiofónica actual. Por lo tanto, los futuros profesionales se convierten durante el desarrollo de la asignatura en creativos, orientados y evaluados de manera continuada por las profesoras y por expertos profesionales en activo, que los asesoran sobre las necesidades reales y las características vigentes de la publicidad. Este principio sigue la línea de lo planteado por Sabán (2000, p. 93) y Mertens (1998) sobre las principales características de lo que sería un programa de capacitación por competencias. Según estos autores, “Las competencias que los alumnos tendrán que cumplir son cuidadosamente identificadas, verificadas por expertos locales y de conocimiento público". El proceso docente se basa así en la orientación sobre dinámicas emprendedoras y versátiles de actuación en el mundo laboral de la publicidad en radio.

3. Principio de adquisición de competencias comunicativas según las necesidades profesionales. Este principio, basado en una habilidad esencial de la EEES, busca desarrollar estas competencias mediante el fomento de una cultura del debate fundamentada en el pensamiento crítico y analítico. Por lo tanto, los 
estudiantes se convierten en comunicadores que tienen que demostrar su capacidad de asimilación de conocimientos y su competencia en comunicación escrita y oral. El proceso docente se basa aquí en la adquisición de unas competencias comunicativas esenciales en el futuro trabajo de un publicitario radiofónico. "Nos interesa proponer un cambio de paradigma, observando la educación desde su práctica y elaborando teoría a partir de su reflexión. Es este un posicionamiento basado en la teoría crítica de la enseñanza, que se distancia de una visión meramente tecnológica" (Bergero y Esnaola, 2006).

4. Principio de la aplicabilidad de las nuevas tecnologías de la información y las comunicaciones al contexto profesional. El análisis de la actual industria radiofónica publicitaria ha permitido identificar determinadas necesidades de carácter técnico y tecnológico. Por lo tanto, los estudiantes se convierten en productores y realizadores de varias piezas radiofónicas utilizando los programas de edición de sonido más comunes en las agencias de publicidad y en las emisoras radiofónicas. De este modo, el proceso docente se basa en la aplicabilidad de herramientas tecnológicas utilizadas en el mundo laboral.

Si bien este proyecto es de directa aplicación a las asignaturas de producción y realización de publicidad en radio, también tiene fácil adaptación a cualquier asignatura relacionada con los medios audiovisuales. Se trata, en algunos casos, de la polémica figura del comunicador de los nuevos tiempos:

"El comunicador debe ser una figura polivalente, multimedia, capaz de producir contenidos en diferentes lenguajes y medios. Los anuncios para buscar trabajadores en múltiples campos de la comunicación ya especifican que los aspirantes deben poseer muchas y más variadas competencias que cubran toda la 'cadena de producción'" (Bergero y Esnaola, 2006). 


\section{Elementos pedagógicos del proyecto}

A continuación, se describen los elementos pedagógicos y tecnológicos del proyecto, tomando como referencia los principios y objetivos que se pretenden lograr:

1. Principio de identificación y resolución de problemas profesionales reales. Como se ha explicado, durante el desarrollo de la asignatura los futuros profesionales se convierten en planificadores de dos campañas publicitarias basadas en necesidades y datos de clientes reales. Por lo tanto, en las sesiones presenciales no trabajan la exposición de contenidos sino la asimilación de conocimientos a partir del uso de la plataforma Moodle. En la línea de lo establecido por Saban (2000), "la enseñanza deber ser menos dirigida a exponer temas y más al proceso de aprendizaje de los individuos." Por esta razón, los estudiantes cuentan con material previo a cada clase que han de consultar. El material está compuesto por: podcasts sonoros explicativos de cada tema que, con una duración media de unos diez minutos, pueden ser descargados al móvil; presentaciones multimedia que recogen las ideas básicas de cada tema; sesiones de debate con profesionales del sector y grabaciones sonoras disponibles en la plataforma Moodle.

2. Principio de consecución de resultados dirigidos a la práctica profesional. Los futuros profesionales se convierten durante la sesión en creativos, orientados y evaluados de manera continuada por las profesoras y por expertos profesionales en activo, que los asesoran sobre las necesidades reales y las características vigentes de la publicidad. A tal fin, los estudiantes previamente preparan las sesiones mediante la escucha de un Podcast sonoro de unos diez minutos de duración que resume las ideas principales a tratar en clase y lo complementan con las presentaciones multimedia disponibles en la plataforma Moodle. Las sesiones presenciales se dedican así al análisis de la adaptación de 
los proyectos presentados a las ideas clave de cada tema. Algunas de ellas cuentan con la presencia de profesionales del sector y son grabadas y puestas a disposición de los estudiantes después en la plataforma Moodle. De esta manera, los alumnos asisten a sesiones de asimilación de conocimientos, a sesiones de redacción y grabación de proyectos (redacción y corrección de guiones a través de las actividades y el foro de consulta de la plataforma Moodle y grabación de la locución en los estudios de radio), a sesiones de evaluación en las que los estudiantes realizan una presentación multimedia de los proyectos realizados ante un profesional del sector $\mathrm{y}$, por último, realizan tertulias en los estudios de radio donde comentan las principales ideas de la clase, que son grabadas y puestas a su disposición posteriormente en la plataforma Moodle.

3. Principio de adquisición de competencias comunicativas según las necesidades profesionales. Los estudiantes se convierten en comunicadores que deben demostrar su capacidad de asimilación de conocimientos, su competencia en la defensa de los proyectos finales ante profesionales reconocidos del sector además de mostrar sus habilidades comunicativas escritas en la realización de guiones de publicidad radiofónica y las orales en la locución de las distintas piezas publicitarias. A tal fin, al acabar cada clase, se realizan sesiones de asimilación de conocimientos. Las conclusiones principales y reflexiones finales se ponen en común en formato tertulia radiofónica, grabada en los estudios de radio donde los estudiantes demuestran el conocimiento adquirido pero también su capacidad de exposición oral. Estas tertulias reflexivas se cuelgan posteriormente en la plataforma Moodle. Así mismo, su competencia en comunicación oral la demuestran en las sesiones de grabación de proyectos, donde locutan los guiones elaborados, y en las sesiones finales de evaluación, donde presentan los proyectos ante un profesional del sector. Además de la evaluación del profesional y de los profesores, los estudiantes también valoran 
los proyectos de sus compañeros a través de la herramienta encuesta de la plataforma Moodle. Las competencias en comunicación escrita las trabajan en la redacción de los guiones de los proyectos con un seguimiento y evaluación mediante Moodle y su foro de consulta.

4. Principio de la aplicabilidad de las nuevas tecnologías de la información y las comunicaciones al contexto profesional. Los estudiantes se convierten en productores y realizadores de varias piezas radiofónicas utilizando los programas de edición de sonido más comunes en las agencias de publicidad y en las emisoras radiofónicas. Además, el segundo de los proyectos tiene que adoptar el formato de Podcast sindicado.

La metodología que se aplica en el desarrollo de este proyecto es especialmente activa, analítica y práctica y está basada en la interrelación sistémica de competencias académicas, profesionales y analíticas utilizando varios métodos productivos (fenomenológico, sintético y analógico) y científicos (deductivo, inductivo, analítico y simbólico) para un aprendizaje operativo. Por lo tanto, potencia los ejes procedimental y actitudinal, y conjuga además la actividad individual con la cooperativa (trabajo en equipo).

\section{Desarrollo del proyecto}

Este proyecto se desarrolla durante la actividad docente de la asignatura anteriormente mencionada en las diez semanas en que se encuentra programada en la UPF. Los estudiantes se encuentran divididos en grupos reducidos y cada sesión de clase cuenta con una duración de tres horas. La siguiente tabla ilustra el desarrollo de actividades por cada sesión:

Tabla 1. Programación semanal de la asignatura 


\begin{tabular}{|c|c|c|c|}
\hline Acción & Indicador & $\begin{array}{l}\text { Mecanismos de } \\
\text { coordinación y } \\
\text { seguimiento }\end{array}$ & Recursos previstos \\
\hline $\begin{array}{c}\text { Análisis de la } \\
\text { situación de la } \\
\text { industria radiofónica. } \\
\text { Planteamiento del } \\
\text { problema }\end{array}$ & $\begin{array}{c}\text { Campaña publicitaria } \\
\text { en forma de cuña } \\
\text { radiofónica. }\end{array}$ & $\begin{array}{c}\text {-Corrección de } \\
\text { propuestas } \\
\text { provisionales } \\
\text { (plataforma Moodle) } \\
\text {-Cuestionario de } \\
\text { resolución de } \\
\text { problemas (Hot } \\
\text { Potatoes Quiz -Moodle- } \\
\text { ). }\end{array}$ & $\begin{array}{l}\text {-Informes sobre } \\
\text { audiencias y cobertura } \\
\text { geográfica de las } \\
\text { emisoras de radio } \\
\text {-Brifings de empresas e } \\
\text { instituciones reales } \\
\text {-Tertulia radiofónica }\end{array}$ \\
\hline $\begin{array}{l}\text { Los géneros } \\
\text { publicitarios: la cuña }\end{array}$ & $\begin{array}{l}\text { Planificación de una } \\
\text { cuña publicitaria de } \\
\text { manera individual }\end{array}$ & $\begin{array}{c}\text { Presentación pública, } \\
\text { análisis y corrección } \\
\text { definitiva de } \\
\text { propuestas presentadas }\end{array}$ & $\begin{array}{l}\text {-Podcast sobre la } \\
\text { situación de la } \\
\text { publicidad en radio } \\
\text {-Presentación con las } \\
\text { ideas básicas } \\
\text {-Sesión debate con un } \\
\text { planificador (grabación y } \\
\text { subida al Moodle) }\end{array}$ \\
\hline $\begin{array}{l}\text { El lenguaje y la } \\
\text { redacción en radio }\end{array}$ & $\begin{array}{c}\text { Redacción de guiones } \\
\text { y locución }\end{array}$ & $\begin{array}{l}\text { Corrección de guiones } \\
\text { y locución (plataforma } \\
\text { Moodle y estudios de } \\
\text { radio) }\end{array}$ & $\begin{array}{l}\text {-Podcast sobre lenguaje } \\
\text { radiofónico } \\
\text {-Presentación con las } \\
\text { ideas básicas } \\
\text {-Bases de datos de cuñas } \\
\text { creativas premiadas } \\
\text {-Tertulia radiofónica }\end{array}$ \\
\hline El montaje radiofónico & $\begin{array}{c}\text { Producción y montaje } \\
\text { de la cuña }\end{array}$ & $\begin{array}{l}\text { Corrección de } \\
\text { propuestas } \\
\text { provisionales } \\
\text { (plataforma Moodle) }\end{array}$ & $\begin{array}{l}\text {-Podcast sobre montaje } \\
\text { - Presentación con las } \\
\text { ideas básicas } \\
\text {-Programa de edición de } \\
\text { sonido. } \\
\text {-Bases de datos de sonido }\end{array}$ \\
\hline $\begin{array}{l}\text { Presentación y } \\
\text { evaluación del } \\
\text { proyecto }\end{array}$ & $\begin{array}{c}\text { Audición y defensa } \\
\text { de las cuñas }\end{array}$ & $\begin{array}{c}\text { Corrección de } \\
\text { propuestas definitivas } \\
\text { (presencial) }\end{array}$ & $\begin{array}{l}\text {-Defensa del proyecto } \\
\text { con presentación } \\
\text { multimedia. } \\
\text {-Presencia ante un } \\
\text { creativo } \\
\text { - Tertulia radiofónica }\end{array}$ \\
\hline $\begin{array}{l}\text { Los géneros } \\
\text { publicitarios: el } \\
\text { microespacio }\end{array}$ & $\begin{array}{c}\text { Proyecto de una } \\
\text { campaña publicitaria } \\
\text { en forma de } \\
\text { microespacio }\end{array}$ & $\begin{array}{l}\text { Presentación pública de } \\
\text { propuestas (plataforma } \\
\text { Moodle) }\end{array}$ & $\begin{array}{l}\text {-Podcast sobre el } \\
\text { microespacio } \\
\text { - Presentación con las } \\
\text { ideas básicas } \\
\text {-Recopilación de } \\
\text { microespacios de las } \\
\text { principales empresas e } \\
\text { instituciones }\end{array}$ \\
\hline $\begin{array}{l}\text { La entrevista en un } \\
\text { microespacio }\end{array}$ & $\begin{array}{c}\text { Planificación de un } \\
\text { microespacio } \\
\text { publicitario en equipo }\end{array}$ & $\begin{array}{c}\text { Corrección definitiva de } \\
\text { propuestas } \\
\text { (plataforma Moodle) }\end{array}$ & $\begin{array}{l}\text {-Podcast sobre la } \\
\text { entrevista } \\
\text { - Presentación con las } \\
\text { ideas básicas }\end{array}$ \\
\hline
\end{tabular}




\begin{tabular}{|c|c|c|c|}
\hline & & & $\begin{array}{l}\text {-Simulación de una } \\
\text { entrevista radiofónica }\end{array}$ \\
\hline $\begin{array}{l}\text { El guión y la locución } \\
\text { de un microespacio }\end{array}$ & $\begin{array}{l}\text { Redacción de los } \\
\text { guiones y realización } \\
\text { de entrevistas }\end{array}$ & $\begin{array}{l}\text { Seguimiento de los } \\
\text { contactos y entidades y } \\
\text { realización de } \\
\text { entrevistas }\end{array}$ & $\begin{array}{l}\text {-Grabadoras de sonido } \\
\text {-Tertulia radiofónica }\end{array}$ \\
\hline $\begin{array}{l}\text { El montaje radiofónico } \\
\text { de un microespacio }\end{array}$ & $\begin{array}{l}\text { Producción y montaje } \\
\text { del microespacio }\end{array}$ & $\begin{array}{c}\text { Corrección de } \\
\text { propuestas } \\
\text { provisionales } \\
\text { (plataforma Moodle) }\end{array}$ & $\begin{array}{l}\text { - Programa de edición de } \\
\text { sonido. } \\
\text { - Bases de datos de } \\
\text { sonido }\end{array}$ \\
\hline $\begin{array}{l}\text { Presentación y } \\
\text { evaluación del } \\
\text { proyecto }\end{array}$ & $\begin{array}{l}\text { Audición y defensa } \\
\text { de los microespacios }\end{array}$ & $\begin{array}{c}\text { Corrección de } \\
\text { propuestas definitivas }\end{array}$ & $\begin{array}{l}\text {-Defensa del proyecto y } \\
\text { presentación multimedia } \\
\text {-Presencia de expertos } \\
\text { profesionales del sector } \\
\text {-Encuesta de evaluación }\end{array}$ \\
\hline
\end{tabular}

Fuente: Elaboración propia.

\section{Evaluación del proyecto}

Todas las actividades organizadas en el proyecto de la asignatura son evaluadas con indicadores cualitativos y cuantitativos, en una dinámica de evaluación continua de la que los estudiantes son partícipes desde el inicio. En la siguiente tabla se resume el proceso de evaluación:

Tabla 2. Evaluación de la asignatura

\begin{tabular}{|c|c|c|c|}
\hline Principio & Objetivo & $\begin{array}{l}\text { Indicadores } \\
\text { cualitativos }\end{array}$ & $\begin{array}{l}\text { Indicadores } \\
\text { cuantitativos }\end{array}$ \\
\hline \multirow{3}{*}{$\begin{array}{l}\text { Identificación y } \\
\text { resolución de } \\
\text { problemas } \\
\text { profesionales reales }\end{array}$} & \multirow{3}{*}{$\begin{array}{l}\text { Reconocer las características del } \\
\text { medio radiofónico como soporte } \\
\text { publicitario identificando y } \\
\text { resolviendo sus actuales } \\
\text { limitaciones en la planificación de } \\
\text { productos publicitarios }\end{array}$} & $\begin{array}{c}\text { Grado de } \\
\text { identificación de } \\
\text { necesidades }\end{array}$ & 1 punto \\
\hline & & $\begin{array}{l}\text { Grado de adaptación } \\
\text { al público objetivo }\end{array}$ & 1 punto \\
\hline & & $\begin{array}{l}\text { Grado de resolución } \\
\text { de limitaciones }\end{array}$ & 1 punto \\
\hline $\begin{array}{l}\text { Consecución de } \\
\text { resultados dirigidos } \\
\text { a la práctica } \\
\text { profesional }\end{array}$ & $\begin{array}{l}\text { Realizar una cuña publicitaria de } \\
\text { manera individual y de un } \\
\text { microespacio en equipo aportando } \\
\text { resultados innovadores y }\end{array}$ & $\begin{array}{l}\text { Grado de adaptación } \\
\text { a las características } \\
\text { sonoras del medio } \\
\text { radiofónico }\end{array}$ & 1 punto \\
\hline
\end{tabular}




\begin{tabular}{|c|c|c|c|}
\hline & originales & $\begin{array}{l}\text { Grado de creatividad } \\
\text { y originalidad de la } \\
\text { propuesta }\end{array}$ & 1 punto \\
\hline \multirow{3}{*}{$\begin{array}{l}\text { Adquisición de } \\
\text { competencias } \\
\text { comunicativas }\end{array}$} & \multirow{3}{*}{$\begin{array}{l}\text { Redactar y locutar los guiones de } \\
\text { la cuña y el microespacio y } \\
\text { defensa de las propuestas en } \\
\text { exposición y debate público. }\end{array}$} & $\begin{array}{l}\text { Grado de adaptación } \\
\text { a las peculiaridades } \\
\text { del lenguaje } \\
\text { radiofónico }\end{array}$ & 1 punto \\
\hline & & $\begin{array}{c}\text { Grado de } \\
\text { competencia en la } \\
\text { interpretación }\end{array}$ & 1 punto \\
\hline & & $\begin{array}{c}\text { Grado de } \\
\text { competencia en la } \\
\text { argumentación de la } \\
\text { defensa } \\
\end{array}$ & 1 punto \\
\hline \multirow{2}{*}{$\begin{array}{c}\text { Aplicación de } \\
\text { nuevas tecnologías }\end{array}$} & \multirow{2}{*}{$\begin{array}{l}\text { Aplicar los recursos del montaje } \\
\text { radiofónico a la producción y } \\
\text { realización de la cuña y el } \\
\text { microespacio utilizando los } \\
\text { programas de edición de sonido }\end{array}$} & $\begin{array}{l}\text { Grado de calidad } \\
\text { técnica de la pieza }\end{array}$ & 1 punto \\
\hline & & $\begin{array}{c}\text { Grado de aplicación } \\
\text { de los recursos } \\
\text { técnicos. }\end{array}$ & 1 punto \\
\hline \multicolumn{3}{|c|}{ Total } & 10 puntos \\
\hline
\end{tabular}

Fuente: Elaboración propia

\section{Ventajas del proyecto}

En relación al enfoque actual, esta propuesta busca modificar su planteamiento en un intento de fortalecer el proceso de adquisición de las competencias necesarias para que los estudiantes sean capaces de desarrollarse en el contexto profesional de forma eficiente. Por lo tanto, las ventajas más destacadas son:

1. Fortalecimiento de la participación activa del estudiante. Con respecto al modelo actual, el estudiante pasa a adoptar un papel más activo, no sólo realizando la búsqueda, escucha y revisión previa de los temas, sino a través de su participación en las sesiones de debate con los profesionales del sector y en las tertulias radiofónicas, además de en la evaluación final de los proyectos. Por lo tanto, posibilita el desarrollo de las capacidades de autonomía y creatividad y 
de actitudes de exploración y curiosidad. Una estrategia acorde con lo establecido por otros autores en campos similares.

"Las prácticas favorecen el desarrollo de estrategias afectivo-emocionales, motivadas, de algún modo, por la cercanía física del docente y la emotividad que emana de los medios. Esta proximidad revierte en una mayor confianza del alumno en sus posibilidades y le motiva para la superación. La afectividad fortalece en el estudiante su voluntad de "querer aprender" (Alonso y López, 1999).

2. Aplicación de un proceso de aprendizaje guiado y basado en proyectos. El modelo que se pretende implantar incorpora un aprendizaje que tiene más posibilidad de resistencia al olvido porque confronta objetivos significativos para el estudiante. Por lo tanto, facilita la transferencia y la retención de la información y el desarrollo de competencias transversales de trabajo en equipo, cooperación, eficiencia y agilidad en la toma de decisiones.

3. Utilización de una metodología de enseñanza innovadora y motivadora. Con la metodología que se busca implantar, los estudiantes constatan experimentalmente los resultados de sus decisiones y actitudes sobre casos reales, de tal manera que pueden asimilar y reflexionar sobre el proceso, a través de la resolución de problemas, el análisis de casos, el debate y el aprendizaje colaborativo, entre otros. Todo ello hace que el grado de implicación y motivación sea más elevado.

4. Refuerzo de la evaluación continua e incremento participativo. En este nuevo modelo se refuerza la evaluación continua del estudiante incorporando la valoración de los profesionales del sector además de sus apreciaciones sobre el 
trabajo realizado por sus compañeros. Por lo tanto, se incrementa el grado de asimilación de la información, sustentado en la vía reflexiva.

5. Acercamiento a la industria radiofónica. El acercamiento a la industria radiofónica, gracias a la participación activa en el proceso de aprendizaje de profesionales del sector, permite al estudiante tener una visión más directa de los problemas del mercado publicitario radiofónico y despertar así su espíritu crítico, ajustando sus propuestas con nuevas ideas y formas de proceder en la práctica que respondan a las necesidades reales.

6. Uso de las nuevas tecnologías.

7. Alineamiento con los objetivos estratégicos del EEES. Se trata de un proyecto que aplica una metodología docente que favorece el aprendizaje activo y el trabajo en equipo.

\section{Resultados e impacto del proyecto}

Mediante la reconversión de la asignatura planteada en este proyecto se espera fortalecer la formación académica del estudiante con una sólida y cualificada preparación profesional y tecnológica, ofreciéndole una visión real y estructurada del mundo laboral como base del proceso de enseñanza y aprendizaje universitario. El resultado derivado se centra en conseguir una adaptación gradual a su actividad laboral, a través de la generación de conciencia, el sentido de responsabilidad y la vivencia profesional, la cooperación organizacional y el compromiso social.

En este mismo sentido, la industria radiofónica y sus profesionales se integran en una dinámica universitaria con el resultado esperado de conseguir una vinculación y retroalimentación directa entre ambos que les permita conocer de primera mano la 
creatividad y las propuestas de las nuevas generaciones y de este modo trabajar para la innovación creativa en sus respectivos ámbitos. Esto permitirá despertar en los estudiantes el espíritu crítico ante la situación del mercado publicitario radiofónico, generando nuevas ideas y formas de proceder en la práctica a través de un ejercicio analítico y aplicativo, además de aportarles un elevado valor y componente motivacional.

Por otro lado, tanto la estructura aplicada en este proyecto como su base en el uso de recursos tecnológicos, permitirá el desarrollo de los conocimientos pertinentes, las competencias profesionales y las habilidades operativas necesarias para conseguir facilitar la inserción del estudiante en el mundo laboral, garantizando sus destrezas y habilidades en la producción y realización radiofónica. Además, la metodología aplicada en este aprendizaje operativo y procedimental permitirá desarrollar la capacidad y el gusto por aprender mediante dinámicas motivadoras que puedan despertar en el estudiante la curiosidad de saber, el deseo de aprender y el gusto por trabajar en equipo con el resultado esperado de incrementar la tasa de rendimiento del alumnado.

Junto con esto, la planificación de actividades y evaluaciones de este proyecto ofrecerá una visión real y crítica de la situación de la publicidad en el medio radiofónico con la intención de desarrollar sus aptitudes críticas, reflexivas y constructivas alrededor de la situación de la industria publicitaria radiofónica.

Finalmente, el fortalecimiento de la formación en una materia débil en la estructura universitaria como es la publicidad radiofónica permitirá revertir en una mejora de la calidad de los procesos productivos y creativos profesionales. Por lo tanto, la propuesta presenta una dimensión integradora, interdisciplinaria y transversal sobre varias materias de los grados de comunicación (Periodismo, Comunicación Audiovisual y Publicidad y Relaciones Públicas). 


\section{Conclusiones y líneas de trabajo}

Sin una adecuada formación radiofónica en los estudios de publicidad, no habrá profesionales que sepan crear contenidos publicitarios adaptados a las características del medio, con lo cual no podrá superarse la crisis de la industria radiofónica. Por lo tanto, este proyecto trata de reaccionar ante la actual situación de crisis de la publicidad en radio, detectada y denunciada por varios investigadores de la materia. Además de las diferentes propuestas innovadoras puestas en marcha en la asignatura y descritas a lo largo del presente texto, aun queda mucho trabajo por hacer. Si pretendemos seguir trabajando en la formación de profesionales y en la dignificación de la actividad profesional en nuestro campo profesional debemos mirar al futuro y afrontar nuevos retos entre los que formulan las siguientes líneas de actuación:

1. Elaborar estrategias y recursos que consideren los diferentes estilos de aprendizaje de los estudiantes y que favorezcan su participación y motivación. Se trata de formar ciudadanos seguros "bien informados y profundamente motivados, provistos de un sentido crítico y capaces de analizar los problemas de la sociedad, buscar soluciones para los problemas que se planteen a la sociedad, aplicar éstas y asumir responsabilidades sociales" (Jiménez y Moncholí, 2009, p. 147).

2. Integrar los recursos informativos y comunicativos para la docencia de una asignatura, o conjunto de asignaturas, con las posibilidades de comunicación que hay en el aula Global, y de la Web 2.0 en el marco del aula Global. "Con la emisión del trabajo práctico en la red, algunas universidades completan el esquema comunicativo, fijan los conocimientos de los estudiantes y les permiten experimentar conceptos profesionales". Jiménez y Moncholí, 2009 (p. 144). 


\section{Bibliografía}

ALONSO, J.; LÓPEZ, G. (1999): Efectos motivacionales de las actividades docentes en función de las motivaciones de los alumnos en Pozo I. y Monereo, C. (coord.): El aprendizaje estratégico. Enseñar a aprender desde el currículo. Aula XXI Santillana. Madrid.

ANECA (2004): Libro Blanco. Títulos de grado en comunicación. Agencia Nacional de Evaluación de la Calidad y Acreditación ANECA. Consultado el 20 de agosto de 2011. Disponible en:

www.aneca.es/media/150336/libroblanco_comunicacion_def.pdf.

BARBEITO, M.; FAJULA, A. (2005): La radio publicitaria: el peso del inmovilismo en Quaderns del CAC, $\mathrm{n}^{\circ} 22$.

BERGERO, I.; ESNAOLA, G. (2006): Nuevos retos para la formación universitaria de los comunicadores: ¿Cómo se construye el aprendizaje mediado por las TICs? en Revista Iberoamericana de Educación, no 39. Consultado el 24 de agosto de 2011. Disponible en:

www.rieoei.org/1449.htm.

GARCÍA, F. (2008): La publicidad en radio: imágenes de baja intensidad retórica. Universidad de Vigo. Pontevedra.

JIMÉNEZ MARTÍN, S.; MONCHOLI, M. A. (2009): La adecuación de la docencia de la radio y televisión a los postulados de Bolonia en Revista Icono14, $\mathrm{n}^{\circ}$ 14. Consultado el 24 de agosto de 2011. Disponible en:

www.icono14.net.

MERTENS, L. (1998): La gestión por competencia laboral en la empresa y la formación profesional. OEI. Madrid.

MONCLÚS, A.; SABÁN, V. (2008): La enseñanza en competencias en el marco de la educación a lo largo de la vida y la sociedad del conocimiento en Revista Iberoamericana de 
Educación. Monográfico Infancias y Escuelas, $n^{\circ}$ 48. Consultado el 24 de agosto de 2011. Disponible en:

www.rieoei.org/rie47a08.htm.

PROYECTO ICOD (2005): Competencias del comunicador digital en Seminario ICOD. Universidad de Beira Interior. Portugal. Consultado el 24 de agosto de 2011. Disponible en:

www.icod.ubi.pt/es/es_competencias_tendencias.html.

RODERO, E. (2008): Publicidad en Radio: publicidad sí, pero no radiofónica en Revista Área Abierta, $\mathrm{n}^{\mathrm{O}} 20$.

SABÁN, C. (2000): Dimensiones actuales de la formación y la función de las competencias en Monclús, A. (coord.): Formación y empleo: enseñanza y competencias. Comares. Granada.

VÁZQUEZ, M. (2001): La infravaloración publicitaria del medio radio en Revista Latina de Comunicación Social, nº 37.

WESSELINK, R; LANS, T; MULDER, M; BIEMANS H. (2003): Competence-based education. An example from vocational practice. Education and Competence Studies. Wageningen University and Research Center. The Netherlands. Consultado el 24 de agosto de 2011. Disponible en:

www.voced.edu.au/content/ngv14623.

NÍHIL ÓBSTAT. IMPRIMÁTUR 